\title{
Service restoration in distribution networks using combination of two heuristic methods considering load shedding
}

\author{
Meysam GHOLAMI ${ }^{1}$, Jamal MOSHTAGH ${ }^{1}$, Naji GHADERNEJAD ${ }^{2}$
}

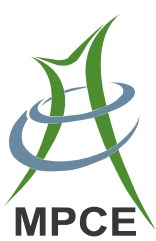

\begin{abstract}
Two heuristic methods are proposed to find an effective and fast solution in modern power distribution networks. For solving the service restoration problem in distribution networks, switch selection indices based on an analytic approach and a practicable heuristic graph-based method are given. The formulation of the problem includes four different objective functions: 1) maximizing the total load restored; 2) minimizing the number of switching operations; 3) maximizing the top priority restored load; 4) minimizing load shedding. A suitable evaluation of switch indices is used for all candidate tie switches (TSs) in the network to find the best solution and decrease the number of switching operations. A new graph-based approach is utilized for finding the best sectionalizes switch (SS) and minimizing the voltage drop. The accuracy and the validity of the approach are tested in two standard electrical distribution networks. The results of the approach are used for IEEE 69-bus and IEEE 119-bus test case.
\end{abstract}

Keywords Service restoration, Modern distribution networks, Smart grid, Switch indices, Graph-based method

CrossCheck date: 2 February 2015

Received: 28 October 2013/Accepted: 16 June 2015/Published online: 9 July 2015

(C) The Author(s) 2015. This article is published with open access at Springerlink.com

$\bowtie$ Jamal MOSHTAGH

jamal.moshtag79@gmail.com

1 Department of Electrical Engineering, University of Kurdistan, Kurdistan, Iran

2 West Regional Electrical Company, Kermanshah, Iran

\section{Introduction}

In each modern electrical distribution network, increasing reliability is considered as one of the most important tasks for electrical distribution companies. With the significant extension of modern power distribution networks in the world, the possibility of the fault occurrence in the network and the consequent blackout for one or more areas are increased. The frequency and the duration of blackouts influence both network reliability and consumer's satisfaction. In order to maintain the consumer's satisfaction and to increase network reliability, the fast restoration of the power supply to out-of-service un-faulted area is required. Due to the high number of switches, feeders and branches in typical distribution systems, it is not easy to restore an outof-service area solely depending on the past experiences of human operators [1]. Therefore, with the advent of computers, changes in technologies and incorporation of smart grid technologies, decreasing the out-of-service areas is possible. Achieving fast service restoration, reducing operators' errors and in consequence increasing network reliability and consumer's satisfaction are the important advantages of restoration in the smart grid. In fact, the service restoration problem is to find a suitable configuration by transferring the loads in out-of-service un-faulted areas to neighboring feeders after the fault occurrence. In each service restoration plan, there exist several issues to be considered. The main objective of service restoration plan is to maximize the safety of out-of-service loads that must be restored. Service restoration is implemented by changing switch state, therefore, the time taken by the service restoration depends on the number of switching operations. So, the number of switching operations should be controlled to be at the minimum possible rate. In every distribution network, there are some consumers that have high priority 
(such as hospitals, police stations, and fire stations, etc.). In service restoration plan it must be considered that the supply must be restored to the consumers of highest priority. The most important constraint in each distribution network is its radial structure due to various reasons, such as ease of detecting the fault location, fault isolation and simply coordination of protective devices. During the service restoration, if the structure in the network changes, the radial structure constraint must be considered. Bus voltage, line current and element loading also change during the service restoration plan, therefore, it is important if these constraints cross their operational limits. Consumers' satisfaction closely depends on the interruption frequency and the duration. Therefore, for finding a quick solution, the restoration plan runtime must be minimized.

In past years, many methods have been proposed to find a solution for the restoration problem from different perspectives. In [2-6], the heuristic method is used to solve service restoration problem. In [2], comparative studies for four modern heuristic algorithms (reactive tabu search, tabu search, parallel simulated annealing, and genetic algorithms) are presented for the service restoration. In [6], a new heuristic graph-based approach is proposed for solving the service restoration problems in distribution networks. In [7, 8], combination methods are applied. In [7], objective functions have been modeled with the fuzzy set, and then an optimization problem is solved by the genetic algorithm. In [8], the method includes the fuzzy multi criteria evaluation and the grey relational analysis. In $[9,10]$, a restoration problem in distribution networks with dispersed generation (DG) is implemented. In [9], the maximum power supply range of DGs is transformed into a 0-1 Knapsack problem and used branch-and-interface method to solve the restoration problem. In [11], a quantitative evaluation framework is presented for ranking restoration plans with their performance indexes, using the analytical hierarchy process-based fuzzy-grey approach. In [12], mathematical programming is presented to reconfigure the network un-faulted section of the system. In [13], reliability assessment of complex radial distribution network systems is presented. In [14], service restoration with load curtailment of in-service customers via direct load control is implemented. In [15], a fuzzy decision-making approach is applied to determine the most desirable restoration plan with consideration different practical factors. In [16], fuzzy cause-effect networks are used to model the heuristic knowledge inference involved in the restoration plan, however, fuzzy method does not guarantee the optimal solution. In [17], Petri net combined with a rulebased expert system is used to implement the service restoration plan. In [18], G-net inference mechanism with operation rules is applied and the expert system approach is adopted in [19]. In [20], a novel formulation for service restoration in unbalanced three phase distribution networks is presented. In this paper, two heuristic methods are proposed for running service restoration plans in modern distribution networks.

The paper is organized as follows. Section 2 describes the formulation of a typical restoration problem. Section 3 shows indices for ranking networks' switches. Section 4 presents a graph-based method. Section 5 studies two heuristic algorithms for service restoration performances. Section 6 shows a numerical example to demonstrate the quickness and effectiveness of the proposed methods, and finally the conclusion is drawn in Section 7.

\section{Problem formulation}

Service restoration in distribution networks is formulated as a multi-constraint and multi-objective problem, considering consumer's priority and load shedding implementation. Four different objective functions are considered: maximizing the total load restored, minimizing the number of switching operations, maximizing the top priority restored load, and minimizing load shedding. These objectives are described briefly below.

$$
\begin{aligned}
& \max \sum_{k \in N_{\mathrm{TT}}} L_{k}, \\
& \max \sum_{k \in N_{\mathrm{HP}}} L_{k},
\end{aligned}
$$

$\min N_{\mathrm{SO}}$,

$\min \sum_{\mathrm{LS} \in N_{\mathrm{LP}}} L_{\mathrm{LS}}$

where $L_{k}, N_{\mathrm{TT}}, N_{\mathrm{HP}}, N_{\mathrm{SO}}, L_{\mathrm{LS}}$ and $N_{\mathrm{LP}}$ are the energized load in the network, the restorable total buses, the restorable buses with high priority, the number of switch operation, the total load shedding, and the buses with low priority, respectively.

1) Bus voltage limits:

$V_{\min }<V_{k}<V_{\max }$

where $V_{k}$ is the voltage in $k$ bus, $V_{\min }$ is the minimum acceptable bus voltage and $V_{\max }$ is the maximum acceptable bus voltage.

2) Line current limits:

$I_{\min }<I_{j}<I_{\max }$

where $I_{j}$ is the current in $j$ line, $I_{\min }$ is the minimum acceptable line current and $I_{\max }$ is the maximum acceptable line current.

The amount of the bus voltage and the line current are obtained from the load flow calculation. We must try to maintain the amount of these limits in the restoration process. 
In this study, Two heuristic methods are used based on the switch indices and a graph-based approach for finding the best solution to restore the maximum total consumers in deenergized areas considering the minimum switch operations.

\section{Switch ranking}

The base of the presented methods for the indices is to achieve the objective functions defined in Section 2, considering the minimum voltage drop in the network. Due to the radial structure of distribution networks and long paths in the network, it is possible to form a new structure in which the voltage magnitude for some buses at end of paths is unsatisfactory. Under this condition, the voltage drop should be considered as an important index. In this paper, two switch indices are used for making the best selection. The first and the most important index is $H$ which is proportionate to the voltage drop between the substation bus and the primary side of each candidate tie switch (TS). For each TS, $H_{i}$ is defined as:

$H_{i}=\frac{P_{i} R_{i}+Q_{i} X_{i}}{V}$

where $P_{i}, Q_{i}, R_{i}, X_{i}$ and $V$ are the sum of active loads between the substation bus and primary side of TS $i$ (perunit), the sum of reactive loads between the substation bus and primary side of tie switch $i$ (per-unit), the sum of resistance of lines between the substation bus and primary side of tie switch $i$ (per-unit), the sum of inductance of lines between the substation bus and primary side of tie switch $i$ (per-unit) and the substation voltage, respectively. A 16-bus distribution network is shown in Fig. 1. Supposing that one fault has taken place at point A. Therefore, Area 1 is the downstream un-faulted area and TS1 is one of candidate switches for the implementation of the service restoration. For TS1, $H_{i}$ is obtained from node number 5 and 6 that are proportionate to direction 1 (DIR1). TS3 is another candidate switch for implementing service restoration. For TS3, $H_{i}$ is obtained from node numbers 10 , 12 and 13 that are proportionate to direction2 (DIR2).

The second index is $Z_{\text {path }}$ that is the direction impedance (per-unit) for lines lying in the path between the secondary side of each TS and the end bus in the network. For each TS, the index is defined as:

$Z_{\text {path }}=\sum_{b \in N_{\mathrm{br}}} Z_{b}$

In this regard, $Z_{b}$ is the impedance (per-unit) of branch $b$ and $N_{\text {br }}$ is the lines lying in the path between the secondary side of each TS and the end bus in the network.

As shown in Fig. 1, supposing that one fault has taken place at point B, Area2 is the downstream un-faulted area

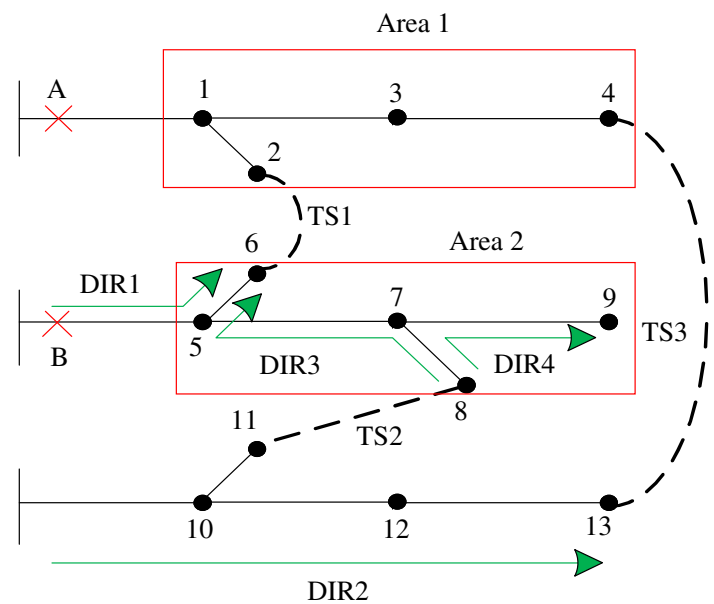

Fig. 1 16-bus distribution network

and TS2 is one of the candidate switches for the implementation of the service restoration. For TS2, $Z_{\text {path }}$ is the impedance of direction3 (DIR3) and direction4 (DIR4). These utilized indices help to both reduce the search space and rank the switches to find the best solution for the service restoration plan. In fact, when the search space is reduced, the runtime of the plan is decreased, thus guarantying the speed of the implementation of the service restoration.

\section{Graph-based method}

The graph-based method is used to solve the problem of service restoration in [6]. For referring to one defect of this reference, the weight factor is determined by the types and characteristics of the branches in the network, not the entire network characteristics. This paper attempts to apply some changes to the method used in [6] in order to obtain good results. Before describing this method to find the minimum voltage drop in the tree (network), we define three groups. For any node (bus) belonging to the graph (network), the following definitions are valid:

1) Sub-graph $D_{\mathrm{H}}$ consists of the nodes that have already been added to the tree.

2) Sub-graph $D_{\mathrm{N}}$ consists of the nodes that have not been added to the tree.

3) Sub-graph $B$ consists of the branches that can be connected to the nodes of sub-graph $D_{\mathrm{H}}$.

When one branch of the tree with minimal weight is selected in each iteration, one node (sending side of branch or node $S$ ) of the branch is in sub-graph $D_{\mathrm{H}}$ and the other node (receiving side or node $R$ ) is in sub-graph $D_{\mathrm{N}}$. Therefore, node $\mathrm{R}$ is added to the sub-graph $D$ and deleted from the sub-graph $D_{\mathrm{N}}$, as shown in Fig. 2. This approach 


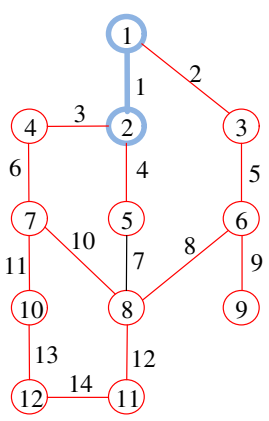

(a) Iteration 1

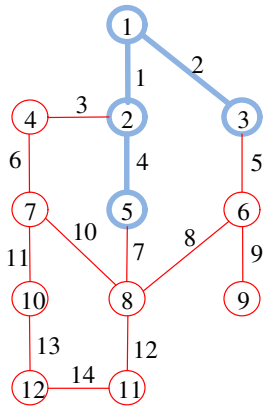

(c) Iteration 3

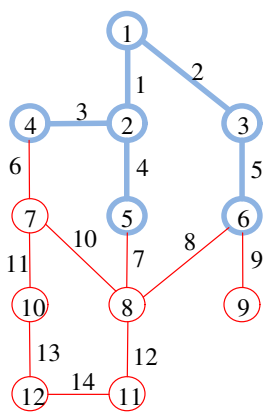

(e) Iteration 5

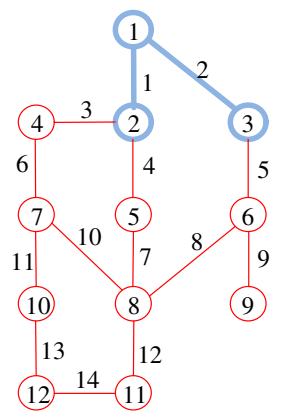

(b) Iteration 2

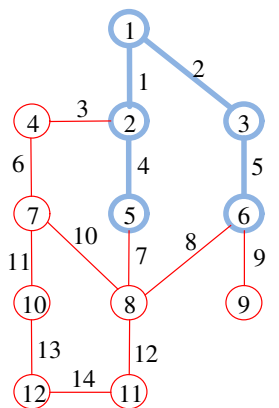

(d) Iteration 4

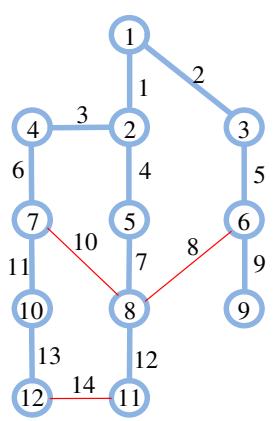

(f) Final structure
Fig. 2 Example of a graph-based method

is used to find the best sectionalizes switch (SS) after finding the best TS. The evaluation of the weight coefficient is described as follows:

$W=X+\sum_{b \in N_{\mathrm{br}}} Z_{b}$

where $X$ is the branch state ( 0 for close branches and inf for open branches); $Z_{b}$ is the impedance of the branch $b$ and $N_{\text {br }}$ is the branches lying in the path between the secondary node (node $R$ ) of the branch in sub-graph $D_{\mathrm{N}}$ and the substation node.

The starting state and the major steps in the first iteration are described as follows:

Starting state:

$\{1\} \in D_{\mathrm{H}}$

$\{2,3,4,5,6,7,8,9,10,11,12\} \in D_{\mathrm{N}}$

$\{1,2\} \in B$
The branches 1 and 2 (in sub-graph B) are between subgraphs $D_{\mathrm{H}}$ and $D_{\mathrm{N}}$.

The first iteration (selection of branch with minimum weight) is as shown in Fig. 2a.

Weight coefficients for branches 1 and 2:

$W_{1}=X_{1}+Z_{1} \quad$ and $\quad W_{2}=X_{2}+Z_{2}$

Therefore, the branch with the minimum of $W$ is selected (supposing that the coefficient is $W_{1}$ ).

Updating sub-graphs:

$$
\begin{aligned}
& \{1,2\} \in D_{\mathrm{H}} \\
& \{3,4,5,6,7,8,9,10,11,12\} \in D_{\mathrm{N}} \\
& \{2,3,4\} \in B
\end{aligned}
$$

Updating $D_{\mathrm{H}}, D_{\mathrm{N}}$ and $B$ is done for the next iteration. When sub-graph $D_{\mathrm{N}}$ gets empty, the iteration procedure is finished. In order to maintain the radial structure of the network, all branches belonging to sub-graph B should be opened after the last iteration. The final structure of the network is shown in Fig. $2 \mathrm{f}$.

$$
\begin{aligned}
& \{1,2,3,4,5,6,7,8,9,10,11,12\} \in D_{\mathrm{H}} \\
& \{\varphi\} \in D_{\mathrm{N}} \\
& \{8,10,14\} \in B
\end{aligned}
$$

To achieve a better understanding of the use of the graphbased method technique, service restoration simulation for a graph-based method is shown Fig. 3. Following the fault occurrence at point A, a number of the loads get deenergized, and the network gets ready to form a new structure for implementing service restoration plan. If two switches (TS3 and TS4) are obtained from the previous section for performing the service restoration, a loop is created in the network. So, an SS switch should be opened to maintain the radial structure of the network. This switch must be selected in such a way as to cause the minimal magnitude of the voltage drop in the network. In this case, before closing the switches TS3 and TS4, the initial conditions for the subgraphs are determined according to the following state.

$\{27,29\} \in D_{\mathrm{H}}$

$\{24,25,26,28,30\} \in D_{\mathrm{N}}$

$\{27-26,29-28\} \in B$

\section{Service restoration algorithm}

To describe the problem of multi-objective service restoration in distribution networks, the fault scenario should be described. When a short-circuit fault occurs on a feeder, the circuit breaker at the outset of feeder operates to clear the fault. All boundary line switches operate to isolate the faulted area. The circuit breaker of the feeder is then 


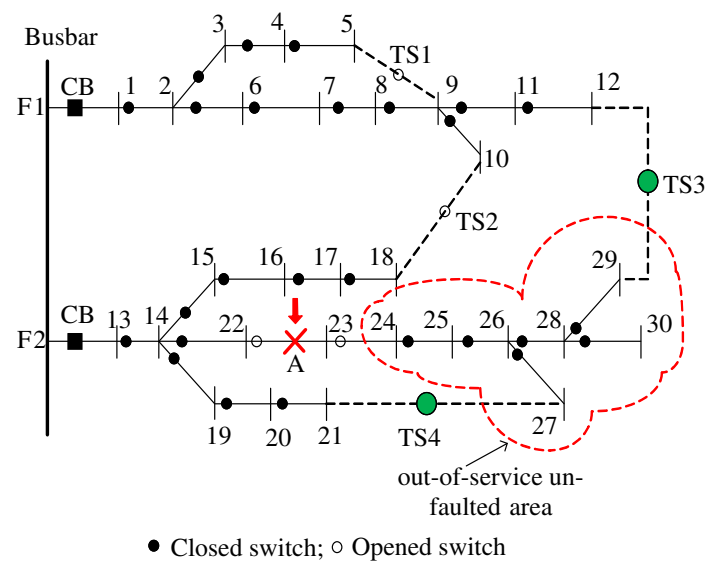

Fig. 3 Service restoration simulation for graph-based method

closed to restore the upstream consumers. For the downstream area, the switch indices for the best switch selection are implemented based on the first heuristic approach (switch index). The proposed approach is calculated and implemented quickly, using automated switches in the distribution network. Four objective functions are considered in the following order: 1) maximizing the amount of total load to be restored; 2) minimizing the number of the switching operations; 3) considering consumers' priority; 4) minimizing total load shedding. The fault location is selected to demonstrate the effectiveness of the proposed algorithm. The proposed algorithm is described as follows:

1) Isolating the fault;

2) Creating a list of the candidate tie switches;

3) Selecting one TS according to the first proposed method in Section 3, implementing the load flow, and studying the network constraints. If no constraint violation exists, go to step 5;

4) Selecting next the TS according to the first proposed method in Section 3, selecting the respective SS according to the graph-based method, implementing the load flow, and studying the network constraints. If no constraint violation exists go to step 5; otherwise, repeat this step;

5) Recognizing the best service restoration plan.

This paper uses the fast load flow technique [21] for fast service restoration performance. When a fault occurs in the distribution network, the fault line, the sending and receiving bus sides are detected. The adjacent buses and lines in all directions are wended sequentially to clear the fault; the first circuit breaker in these directions is found and starts operating. Therefore, the feeder in which the fault has occurred and its circuit breaker are detected. For isolating the fault, the adjacent buses and lines in each direction are wended sequentially. The first switch in each direction is found and starts operating. Therefore three areas are formed in the network: Firstly, the upstream outof-service area is first restored by closing circuit breaker of the feeder. Secondly, the damaged area must been repaired. Finally, the downstream un-faulted area is transferred to the neighboring feeders according to the proposed algorithms. Before applying the method, we must define switch (TS and SS) candidates for the first algorithm. All tie switches connected to the out-of-service un-faulted area are the tie switch candidates for service restoration implementation. All sectionalize switches in the out-of-service un-faulted area are the sectionalize switch candidates. $H$ and $Z_{\text {path }}$ are obtained for each candidate TS. A weight factor is utilized to transform these two indices into an equivalent single index. The final index is described as:

$F=\alpha H_{i}+\beta \max \left(Z_{\text {path }}\right)$

where $\alpha$ and $\beta$ are two weight factors $(0<\alpha<1$ and $0<\beta<1$ ). The first and the most important index has greater weight factor than the second index. The amount of weight factor can be initialized by PDN operators. $\alpha$ is 0.7 . The $F$ list is formed in an increasing order. One TS operates according to the smallest member of $F$ (the first member of TS list), load flow is calculated, and network constraints are checked. Network constraints have been described in Section 2. If all network constraints are satisfactory, the restoration plan is identified and restoration process is stopped. If an overload or voltage violation exists, load shedding must implemented. After load shedding implementation, if the network constraints are unsatisfactory, the next TS candidate must be operated.

\subsection{Load shedding process}

For implementing load shedding in the network, low priority consumers in out-of-service un-faulted area are identified. The SS is identified which belongs to the lines connected to low priority consumers in this area. The SS list is formed in an increasing order according to the amount of load shedding, and the first candidate for load shedding (minimum load shedding amount) is identified. The load flow calculation is applied to the network and the network constraints are checked. If all network constraints are satisfactory, the restoration plan is identified; otherwise, the next load shedding candidate must be implemented.

\subsection{Next TS selection}

The next TS from the $F$ list is firstly operated. And then there is one direction in the network that creates one loop in the network. For removing this loop, the graph-based method is applied in order to find the best SS in the loop direction. So the best SS is operated for minimizing the voltage drop in the network. After TS and SS operations, 
the load flow calculation is applied to the network. If all network constraints are satisfactory, the restoration plan is identified. If overload or voltage violation exists, the load shedding program must be implemented. If the network constraints are unsatisfactory after the load shedding is implemented, the next TS and SS candidates must be operated.

\section{Numerical examples}

Two different distribution networks are considered to illustrate the effectiveness of the proposed methods. The methods are coded in MATLAB software. When a fault occurs in the network, the protection devices start operating immediately for detecting and isolating the fault. The total numbers of switch operations for isolating the fault and service restoration implementation are calculated. 69-bus and 119-bus distribution networks [22, 23] are considered, as shown in Figs. 4 and 5, respectively. The SSs are considered both in the networks and in the sending side of all lines. Two weight factors $(\alpha$ and $\beta$ ) are 0.7 and 0.3 , respectively. In order to demonstrate the validity and the effectiveness of the proposed service restoration algorithm, the fault simulation is considered in several locations. And two scenarios are considered for the service restoration, respectively. In the first scenario (Scenario A), the service restoration is implemented without load shedding. In the second scenario (Scenario B), the load shedding is considered to implement the service restoration plan.

\subsection{Scenario A}

Tables 1 and 2 show the service restoration results in both 69-bus and 119-bus distribution networks without load shedding implementation. In each network, four points have been considered as the fault locations. Supposing that one fault has taken place in lines 5-6 in the 69-bus distribution network (Case1 in Table 1).

Two switch operations (SS 5-6 and SS 6-7) are required for isolating the fault, and three switch operations (TS 11-43, 50-59 and SS 9-53) are needed for service restoration implementation have been required. This is shown in Table 1. The choice of SS is based on the graph-based method. The minimum voltage of the network in the bus 65 is almost 0.9 p.u.. The runtime of the plan for fault isolation and service restoration implementation is $1.06 \mathrm{~s}$. For Case 2 in Table 1, three switch operations are required to isolate the fault. Thus, the fault location is isolated in all directions. For case 1 in Table 2, two switch operations are required for isolating the fault and one switch operation is required for service restoration implementation. The minimum voltage in the bus 76 is almost 0.9 p.u..

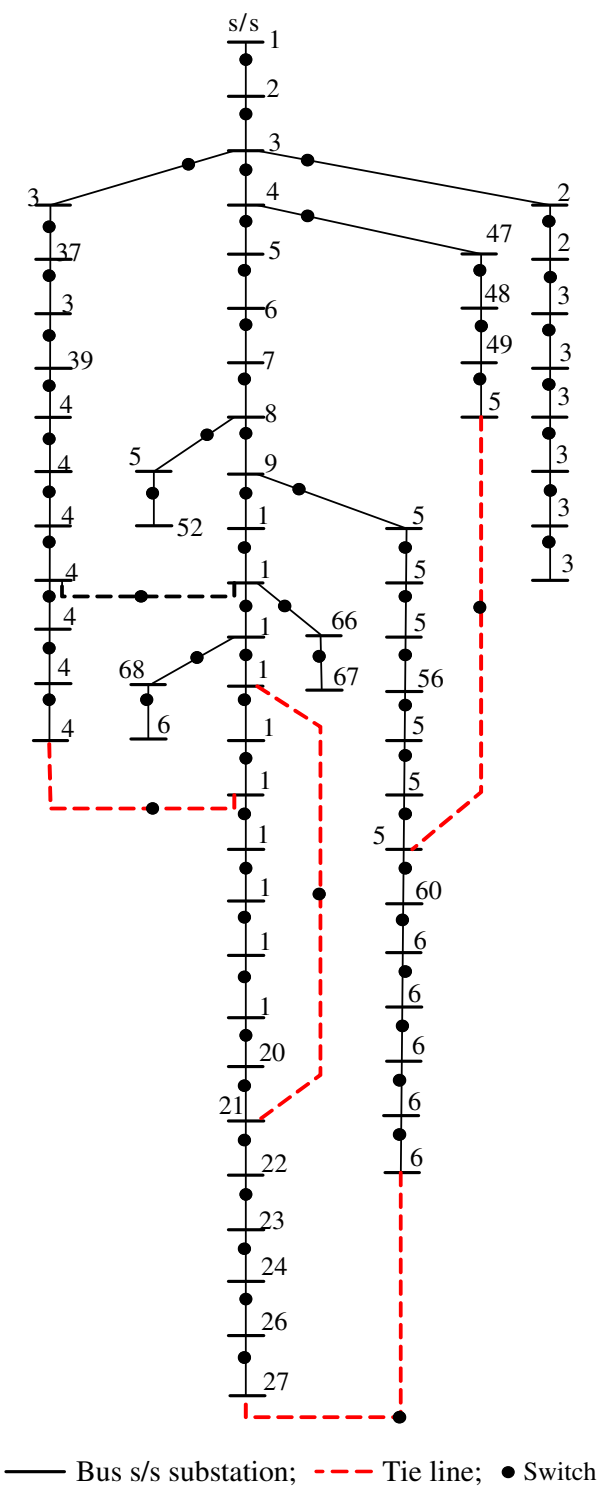

Fig. 4 69-bus distribution network

\subsection{Scenario B}

Tables 3 and 4 display the service restoration results for 69-bus and 119-bus distribution networks considering load shedding plan implementation, respectively. For Case 1 in Table 3, a fault in lines 5-6, one switch operation for implementing service restoration and one switch operation for load shedding process are required. In this case, the number of switching is decreased. The minimum voltage happened in bus 27 is almost 0.95 p.u.. This demonstrates that load shedding process reduces both the number of switch operations and software runtime. For cases 2 and 4 in Table 4, the load shedding process is implemented, too. It is supposed that all the considered switches in both networks are remotely controlled. 


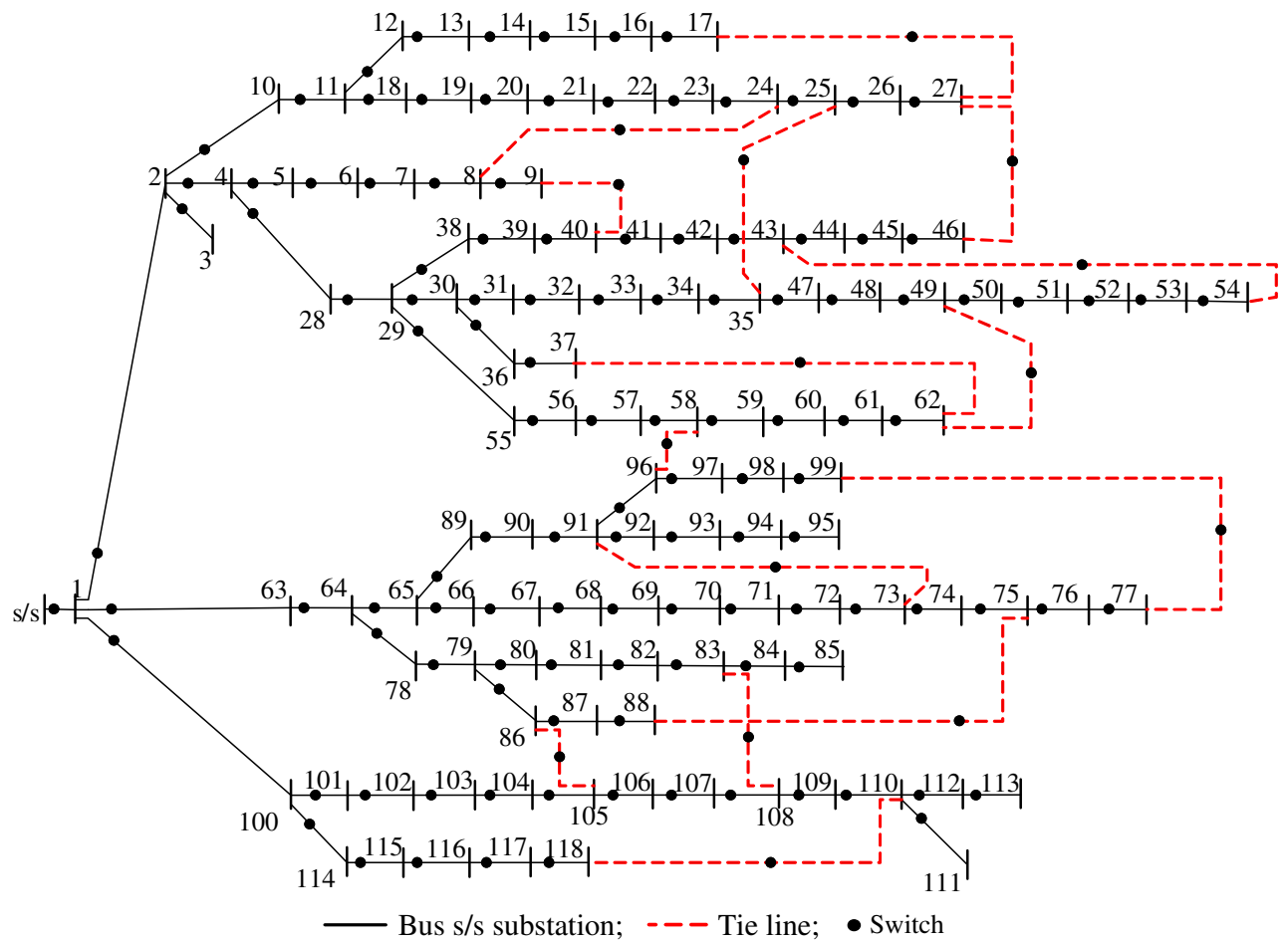

Fig. 5 119-bus distribution network

Table 1 Restoration results for 69-bus network without load shedding

\begin{tabular}{|c|c|c|c|c|c|c|c|}
\hline \multirow[t]{2}{*}{ Case } & \multirow{2}{*}{$\begin{array}{l}\text { Fault } \\
\text { location }\end{array}$} & \multirow{2}{*}{$\begin{array}{l}\text { Switch operation to } \\
\text { fault isolation }\end{array}$} & \multirow{2}{*}{$\begin{array}{l}\text { Switch operation } \\
\text { to restoration }\end{array}$} & \multicolumn{2}{|c|}{ Min. voltage } & \multirow{2}{*}{$\begin{array}{l}\text { Switches to } \\
\text { load shedding }\end{array}$} & \multirow{2}{*}{$\begin{array}{l}\text { Runtime } \\
\text { (s) }\end{array}$} \\
\hline & & & & Bus & Value (p.u.) & & \\
\hline 1 & $5-6$ & $5-6,6-7$ & $11-43,50-59,9-53$ & 65 & 0.9003 & - & 1.06 \\
\hline 2 & $10-11$ & $10-11,11-12,11-66$ & $46-15$ & 65 & 0.9002 & - & 0.54 \\
\hline 3 & $54-55$ & $54-55,55-56$ & $50-59$ & 65 & 0.9010 & - & 0.47 \\
\hline 4 & $18-19$ & $18-19,19-20$ & $13-21$ & 65 & 0.9014 & - & 0.41 \\
\hline
\end{tabular}

Table 2 Restoration results for 119-bus network without load shedding

\begin{tabular}{|c|c|c|c|c|c|c|c|}
\hline \multirow[t]{2}{*}{ Case } & \multirow{2}{*}{$\begin{array}{l}\text { Fault } \\
\text { location }\end{array}$} & \multirow{2}{*}{$\begin{array}{l}\text { Switch operation } \\
\text { to fault isolation }\end{array}$} & \multirow{2}{*}{$\begin{array}{l}\text { Switch operation } \\
\text { to restoration }\end{array}$} & \multicolumn{2}{|c|}{ Min. voltage } & \multirow{2}{*}{$\begin{array}{l}\text { Switches to } \\
\text { load shedding }\end{array}$} & \multirow{2}{*}{$\begin{array}{l}\text { Runtime } \\
\text { (s) }\end{array}$} \\
\hline & & & & Bus & Value (p.u.) & & \\
\hline 1 & $66-67$ & $66-67,67-68$ & $91-73$ & 76 & 0.9021 & - & 0.75 \\
\hline 2 & $31-32$ & $31-32,32-33$ & $49-62,43-54,51-52$ & 34 & 0.9045 & - & 1.75 \\
\hline 3 & 103-104 & $103-104,104-105$ & $110-118$ & 107 & 0.9062 & - & 0.78 \\
\hline 4 & $89-90$ & $89-90,90-91$ & $91-73,58-96,91-96$ & 76 & 0.9022 & - & 1.29 \\
\hline
\end{tabular}

It is necessary to explain that the results (software runtime) are the average of results after 10 times of service restoration implementation.

The most important indices for improving the service restoration plan are the restoration time and the number of switch operations. Table 5 displays the comparison between the results of the proposed method and the results of some of the references (comparing the runtime of service restoration implementation). Table 5 shows the average software runtime for several fault points in the network. According to the results of Table 5, the software runtime is decreased by proposed methods. 
Table 3 Restoration results for 69-bus network considering load shedding

\begin{tabular}{|c|c|c|c|c|c|c|c|}
\hline \multirow[t]{2}{*}{ Case } & \multirow{2}{*}{$\begin{array}{l}\text { Fault } \\
\text { location }\end{array}$} & \multirow{2}{*}{$\begin{array}{l}\text { Switch operation } \\
\text { to fault isolation }\end{array}$} & \multirow{2}{*}{$\begin{array}{l}\text { Switch operation } \\
\text { to restoration }\end{array}$} & \multicolumn{2}{|c|}{ Min. voltage } & \multirow{2}{*}{$\begin{array}{l}\text { Switches to } \\
\text { load shedding }\end{array}$} & \multirow{2}{*}{$\begin{array}{l}\text { Runtime } \\
\text { (s) }\end{array}$} \\
\hline & & & & Bus & Value (p.u.) & & \\
\hline 1 & $5-6$ & $5-6,6-7$ & $11-43$ & 27 & 0.9525 & $57-58$ & 0.91 \\
\hline 2 & $10-11$ & $10-11,11-12,11-66$ & $46-15$ & 65 & 0.9011 & - & 0.48 \\
\hline 3 & $54-55$ & $54-55,55-56$ & $50-59$ & 65 & 0.9074 & - & 0.51 \\
\hline 4 & $18-19$ & $18-19,19-20$ & $13-21$ & 65 & 0.9021 & - & 0.53 \\
\hline
\end{tabular}

Table 4 Restoration results for 119-bus network considering load shedding

\begin{tabular}{|c|c|c|c|c|c|c|c|}
\hline \multirow[t]{2}{*}{ Case } & \multirow{2}{*}{$\begin{array}{l}\text { Fault } \\
\text { location }\end{array}$} & \multirow{2}{*}{$\begin{array}{l}\text { Switch operation } \\
\text { to fault isolation }\end{array}$} & \multirow{2}{*}{$\begin{array}{l}\text { Switch operation } \\
\text { to restoration }\end{array}$} & \multicolumn{2}{|c|}{ Min. voltage } & \multirow{2}{*}{$\begin{array}{l}\text { Switches to } \\
\text { load shedding }\end{array}$} & \multirow{2}{*}{$\begin{array}{l}\text { Runtime } \\
\text { (s) }\end{array}$} \\
\hline & & & & Bus & Value (p.u.) & & \\
\hline 1 & $66-67$ & $66-67,67-68$ & $91-73$ & 76 & 0.9020 & - & 0.69 \\
\hline 2 & $31-32$ & $31-32,32-33$ & $49-62$ & 34 & 0.9273 & $50-51$ & 1.15 \\
\hline 3 & $103-104$ & $103-104,104-105$ & $110-118$ & 107 & 0.9139 & - & 0.76 \\
\hline 4 & $89-90$ & 89-90, 90-91 & $91-73$ & 98 & 0.9212 & $91-92$ & 1.62 \\
\hline
\end{tabular}

Table 5 Comparing results (runtime software) of different methods

\begin{tabular}{llc}
\hline Method & Test case network & $\begin{array}{l}\text { Average of } \\
\text { software } \\
\text { runtime(s) }\end{array}$ \\
\hline Ref. [24] & $\begin{array}{l}\text { Taiwan Power Company } \\
\text { (TPC) distribution network }\end{array}$ & 9.05 \\
Ref. [1] & 32-bus & 12.46 \\
Ref. [20] & 173-bus & 330.44 \\
Proposed & Un-balanced 37-bus & 87.85 \\
algorithms & 69-bus & 0.61 \\
\hline
\end{tabular}

\section{Conclusion}

A fast service restoration in distribution networks is implemented, considering load shedding as a multiple objective function problem. This paper presents two new heuristic methods based on two important indices and a graph-based method. The core of the first proposed algorithm is the voltage drop between candidate TS and the substation bus, and the core of the second algorithm is based on a graph-based method to minimize the voltage drop in the network. Finally, the proposed methods are tested in 69-bus and 119-bus distribution networks on two scenarios (with load shedding and without load shedding). The results show that these methods can be used for solving the service restoration problems in modern distribution networks.

Open Access This article is distributed under the terms of the Creative Commons Attribution 4.0 International License (http:// creativecommons.org/licenses/by/4.0/), which permits unrestricted use, distribution, and reproduction in any medium, provided you give appropriate credit to the original author(s) and the source, provide a link to the Creative Commons license, and indicate if changes were made.

\section{References}

[1] Kumar Y, Das B, Sharma J (2008) Multi-objective, multi-constraint service restoration of electric power distribution system with priority customers. IEEE Trans Power Del 23(1):261-270

[2] Toune S, Fudo H, Genji T et al (2002) Comparative study of modern heuristic algorithms to service restoration in distribution systems. IEEE Trans Power Del 17(1):173-181

[3] Miu K, Chiang H, Yuan B et al (1998) Fast service restoration for large-scale distribution systems with priority customers and constraints. IEEE Trans Power Syst 13(3):789-795

[4] Miu K, Chiang H, McNulty R (2000) Multi-tier service restoration through network reconfiguration and capacitor control for large-scale radial distribution systems. IEEE Trans Power Syst 15(3):1001-1007

[5] Hsu Y, Kuo H (1994) A heuristic based fuzzy reasoning approach for distribution system service restoration. IEEE Trans Power Deliv 9(2):948-953

[6] Dimitrijevic S, Rajakovicb N (2011) An innovative approach for solving the restoration problem in distribution networks. ELSEVIER, Electric Power Syst Res 81:1961-1972

[7] Hsiao Y, Chien C (2000) Enhancement of restoration service in distribution systems using a combination fuzzy-GA method. IEEE Trans Power Syst 15(4):1394-1400

[8] Chen W, Tsai M, Kuo H (2005) Distribution system restoration using the hybrid fuzzy-grey method. IEEE Trans Power Syst 20(1):199-255

[9] Pham T, Bésanger Y, Hadjsaid N (2009) New challenges in power system restoration with large scale of dispersed generation insertion. IEEE Trans Power Syst 24(1):398-406

[10] Kumar V, Kumar R, Gupta I et al (2010) DG integrated approach for service restoration under cold load pickup. IEEE Trans Power Deliv 25(1):398-406 
[11] Chen W (2010) Quantitative decision-making model for distribution system restoration. IEEE Trans Power Syst 25(1):313-321

[12] Butler K, Sarma N, Prasad R (2001) Network reconfiguration for service restoration in shipboard power distribution systems. IEEE Trans Power Syst 16(4):653-661

[13] Li W, Wang P, Li Z et al (2004) Reliability evaluation of complex radial distribution systems considering restoration sequence and network constraints. IEEE Trans Power Del 19(2):753-758

[14] Kleinberg M, Miu K, Chiang H (2011) Improving service restoration of power distribution systems through load curtailment of in-service customers. IEEE Trans Power Syst 26(3):1110-1117

[15] Lee S, Lim S, Ahn B (1998) Service restoration of primary distribution systems based on fuzzy evaluation of multi-criteria. IEEE Trans Power Syst 13(3):1156-1163

[16] Huang C (2003) Multi-objective service restoration of distribution systems using fuzzy cause-effect networks. IEEE Trans Power Syst 18(2):867-874

[17] Chao-Shun C, Lin C, Hung-Ying T (2002) A rule-based expert system with colored petri net models for distribution system service restoration. IEEE Trans Power Syst 17(4):1073-1080

[18] Ke Y (2004) Distribution feeder reconfiguration for load balancing and service restoration by using G-nets inference mechanism. IEEE Trans Power Deliv 19(3):1426-1433

[19] Lee S, Kim K, Nam K et al (1994) Service restoration expert system adopting branch pattern based grouping strategy in distribution systems. Expert Syst Appl Power Syst IV:423-427

[20] Khushalani S, Solanki J, Schulz N (2007) Optimized restoration of unbalanced distribution systems. IEEE Trans Power Syst 22(2):624-630

[21] De Oliveira-De Jesus PM, Alvarez MA, Yusta JM (2013) Distribution power flow method based on a real quasi-symmetric matrix ELSEVIER. Electric Power Syst Res 95:148-159

[22] Savier J, Das D (2007) Impact of network reconfiguration on loss allocation of radial distribution systems. IEEE Trans Power Deliv 22(2):2473-2480
[23] Zhang D, Fu Zh, Zhang L (2007) An improved TS algorithm for loss-minimum reconfiguration in large-scale distribution systems. ELSEVIER, Electric Power Syst Res 77:685-694

[24] Chao MH, Cheng TH, Yung ShW (2011) Evolution of radial basic function neural network for fast restoration of distribution systems with load variations. ELSEVIER, Electrical Power Energy Systems 33:961-968

Meysam GHOLAMI received the B.S. degree in electrical engineering from Urmia University, Iran (2011) and M.S. degree in electrical engineering from Kurdistan University, Iran (2014). Currently, he is relay and network protection expert in Peyman Gharb Company (West Regional Electrical Company). His research interests include distribution network optimization, control of smart grid and power system protection.

Jamal MOSHTAGH received the B.S. degree in Electrical Power Engineering from Sharif University of Technology (Iran) in 1989 and the M.S. degree in the same field from Kh. Nasir University of Technology (Iran) in 1995. He received the PhD degree from the University of Bath (UK) in 2006. At the present, he is the assistant Professor of Electrical engineering Department in University of Kurdistan. His areas of interest include power distribution systems analysis, application of intelligent systems in power distribution systems.

Naji GHADERNEJAD received the B.S. degree in Electrical Power Engineering from Power and Water University of Technology (Iran) in 1998 and the M.S. degree in the Electrical Engineering from Razi University (Iran) in 2015. Now, he is head of supervising department in power protection and DCS in West Regional Electrical Company (Kurdistan Transmission Office) in Iran. His research area focuses on power transformers modeling, wide area protection and control of smart grid. 